\title{
BMJ Open Quality Managing alarm systems for quality and safety in the hospital setting
}

Tita Alissa Bach, Lars-Martin Berglund, Eva Turk

To cite: Bach TA, Berglund L-M, Turk E. Managing alarm systems for quality and safety in the hospital setting.BMJ Open Quality 2018;7:e000202. doi:10.1136/ bmjoq-2017-000202

- Additional material is published online only. To view please visit the journal online (http://dx.doi.org/10.1136/ bmjoq-2017-000202).

Received 8 September 2017 Revised 19 March 2018 Accepted 25 May 2018
Check for updates

Group Technology and Research, Life Sciences program, DNV GL AS, Hovik, Norway

Correspondence to Dr Tita Alissa Bach; tita.alissa.bach@dnvgl.com

\section{ABSTRACT}

Objective To provide an overview of documented studies and initiatives that demonstrate efforts to manage and improve alarm systems for quality in healthcare by human, organisational and technical factors.

Methods A literature review, a grey literature review, interviews and a review of alarm-related standards (IEC 60601-1-8, IEC 62366-1:2015 and ANSI/Advancement of Medical Instrumentation HE 75:2009/2013) were conducted. Qualitative analysis was conducted to identify common themes of improvement elements in the literature and grey literature reviews, interviews and the review of alarm-related standards.

Results 21 articles and 7 publications on alarm quality improvement work were included in the literature and grey literature reviews, in which 10 themes of improvement elements were identified. The 10 themes were categorised into human factors (alarm training and education, multidisciplinary teamwork, alarm safety culture), organisational factors (alarm protocols and standard procedures, alarm assessment and evaluation, alarm inventory and prioritisation, and sharing and learning) and technical factors (machine learning, alarm configuration and alarm design). 26 clinicians were interviewed. 9 of the 10 themes were identified from the interview responses. The review of the standards identified 3 of the 10 themes. The study findings are also presented in a step-by-step guide to optimise implementation of the improvement elements for healthcare organisations.

Conclusions Improving alarm safety can be achieved by incorporating human, organisational and technical factors in an integrated approach. There is still a gap between alarm-related standards and how the standards are translated into practice, especially in a clinical environment that uses multiple alarming medical devices from different manufacturers. Standardisation across devices and manufacturers and the use of machine learning in improving alarm safety should be discussed in future collaboration between alarm manufacturers, end users and regulators.

\section{INTRODUCTION}

Alarming medical devices are designed to generate alarm signals 'to indicate unsatisfactory physiological patient states, unsatisfactory functional states of medical electrical equipment or medical electrical system or to warn the operator of hazards to the patient or operator due to the medical electrical equipment or medical electrical system' $\left({ }^{1},{ }^{\text {p. }}{ }^{8}\right)$. Paradoxically, alarms of medical devices have been suggested to be hazardous to patient safety. ${ }^{2-7}$ There were 566 reports of patient deaths related to monitoring device alarms during 2005-2008 received by the US Food and Drug Administration (FDA) Manufacturer and User Facility Device Experience (MAUDE) ${ }^{8}$ In a more specific example, in 2010 the FDA also received $>2500$ adverse event reports associated with ventilator use, in which about a third of the events indicated an alarm system-related issue. ${ }^{9}$ These numbers are suggested to be an underestimation of an overlooked patient safety issue. ${ }^{2}$ Accordingly, several organisations based in the USA such as the Advancement of Medical Instrumentation (AAMI Foundation), ECRI institute, American Association of Critical-Care Nurses and the Joint Commission have been focusing their efforts to highlight this issue and improve alarm safety in healthcare. $^{4}$

The biggest contributing factor to alarm-related adverse events is suggested to be the excessive amount of alarms in a clinical environment, which can reach up to 942 alarms per day. ${ }^{10}$ This amount of alarms translates to thousands of alarm signals on a single hospital unit. This cacophony of alarms desensitises clinicians, termed 'alarm fatigue', and has become a patient safety concern when clinicians do not respond to clinically critical alarms. ${ }^{2} 4$ Importantly, excessive alarm frequency has been linked to many unfavourable clinician behaviours in attempting to reduce alarm frequency by, for example, disabling or silencing critical alarms, setting inappropriate alarm parameters and reducing alarm volumes, ${ }^{11}$ leading to undesirable patient outcomes. ${ }^{12}$

These blaring alarms are not always clinically critical or actionable. Research suggests that $80 \%-99 \%$ of alarms are false. ${ }^{713-18}$ Alarms are designed by default to be high in sensitivity but low in specificity 'because a manufacturer is much more likely to be held liable for an alarm system that fails to annunciate a clinically significant alarm condition than for any problems caused by annunciating false-alarm conditions' $\left({ }^{19, \text { p. } 207}\right)$. In addition, alarm parameters are usually set to a generalised population 
instead of patient-specific conditions. ${ }^{10}{ }^{17}$ In setting alarm systems in clinical environments, clinicians usually also follow the 'better-safe-than-sorry' logic. ${ }^{20}$

Alarm fatigue has been suggested as the biggest contributor to alarm adverse events, ${ }^{241720}$ and is suggested to occur because humans tend to respond to alarms in the same proportion to their perceived reliability of the alarm system. ${ }^{21} 22$ If alarms are $90 \%$ true, people will respond to them about $90 \%$ of the time, and if the alarms are $10 \%$ true, these alarms will be responded to about $10 \%$ of the time. In addition, clinician response time to alarms increased as non-actionable alarms (true alarms that do not require any clinical interventions or any actions) exposure increased. ${ }^{18}$ Consequently, staff care performance is likely to be compromised by high rates of false and non-actionable alarms, directly impacting patient safety. Importantly, the cacophony of alarms has been shown to delay patient recovery, increase length of stay, ${ }^{7224}$ influence patient satisfaction negatively as reflected in patient surveys ${ }^{724}$ and contribute to patient distress, sleep disturbances, delirium, increased blood pressure and heart rate, and weakened immune system. ${ }^{24}$

Although many studies and initiatives have pinpointed excessive alarms as a patient safety issue,${ }^{425-29}$ studies documenting solutions to this issue are more limited. ${ }^{230}$ This is likely because alarm adverse events are a multifaceted issue involving human, organisational and technical factors. ${ }^{41731}$ The risk for alarm adverse events can increase due to organisational issues such as lack of alarm accountability $^{10} 32$ and lack of agreement on setting alarms, ${ }^{17}$ human issues such as inconsistent clinician response to alarms $^{32}$ and inconsistency in silencing or toning down alarms ${ }^{16}$ and technical issues such as varied urgent alarm tones $^{33}$ and uninformative, confusing alarm signals. ${ }^{34}$ Accordingly, many healthcare organisations continue to struggle with alarm adverse events and are looking for effective ways to reduce them. ${ }^{20} 3135$ To our knowledge, there is minimal research that provides an overview of studies and initiatives that demonstrate efforts to manage and improve alarm systems for quality in healthcare by human, organisational and technical factors. The study aims to provide an overview of studies and initiatives that demonstrate efforts to manage and improve alarm systems for quality and patient safety in the hospital setting by conducting literature and grey literature reviews, interviews with clinicians and a review of alarm-related standards. The research question is, 'How to improve alarm safety in the hospital setting by incorporating human, organisational and technical factors?' Accordingly, findings can be used to support healthcare organisations improving their alarm safety by implementing concrete and practical steps, addressing human, organisational and technical factors.

\section{METHODS}

We conducted literature and grey literature reviews, a review of alarm-related standards and individual interviews with clinicians. For this study, we did not seek any ethical approval by the Norwegian Centre for Research Data because the study did not use or disclose any traceable personal information. ${ }^{36}$

\section{Literature and grey literature reviews}

For the literature search, we used the search engines PubMed and Scopus. Whereas the search of grey literature was conducted using the Google search engine and a snowball technique. The keywords used for the literature and grey literature review were

- "Alarm" AND "Improvement" OR "Effective" OR "Integration" OR "compatibility".

- "Alarm" or "alarms" AND "standard" OR "Recommended practice" AND "Healthcare" OR "health care".

- "Health care" OR "Healthcare" AND "Clinical Alarm".

- "Health care" OR 'Healthcare' AND "Alarm system".

- "Alarm configuration" AND "health care" OR "healthcare".

- "Alarm fatigue".

- "Integrated Alarms".

- "Effective clinical alarms".

- "Patient monitoring system".

- "Patient monitoring system" AND "Standard" OR "Recommended practice".

The inclusion criteria for the reviews were studies or quality improvement work that demonstrated and documented efforts to improve alarms or alarm systems in healthcare, were published between 1 January 2000 to 1 March 2016, written in English and conducted in hospital settings where alarm fatigue mostly occurred.

For the literature review, all authors first individually screened titles and abstracts of articles for eligibility. If at least one author considered an article as relevant, the article was included in full-text reviews. Then, the included full-text articles were screened by authors independently for eligibility. For the grey literature review, all authors searched and reviewed documented improvement work independently for eligibility. Any improvement work that was considered as relevant by at least one author was included in the next round of review. A series of meetings to review and discuss each potential eligible article and documented improvement work were held to decide the final number of publications. Team consensus was used to resolve any disagreements regarding eligibility and relevance. For analysis, common themes of alarm improvement elements were clustered together and used as reference themes for the review of alarm-related standards and interviews. Twenty-one articles were identified as eligible for inclusion in the literature review (figure 1) and seven quality improvement work was identified from the grey literature review (online supplementary appendix 1).

\section{Individual interviews}

Semistructured interviews were conducted by phone and face-to-face and focused on exploring interviewees' perspectives on alarm problems based on 


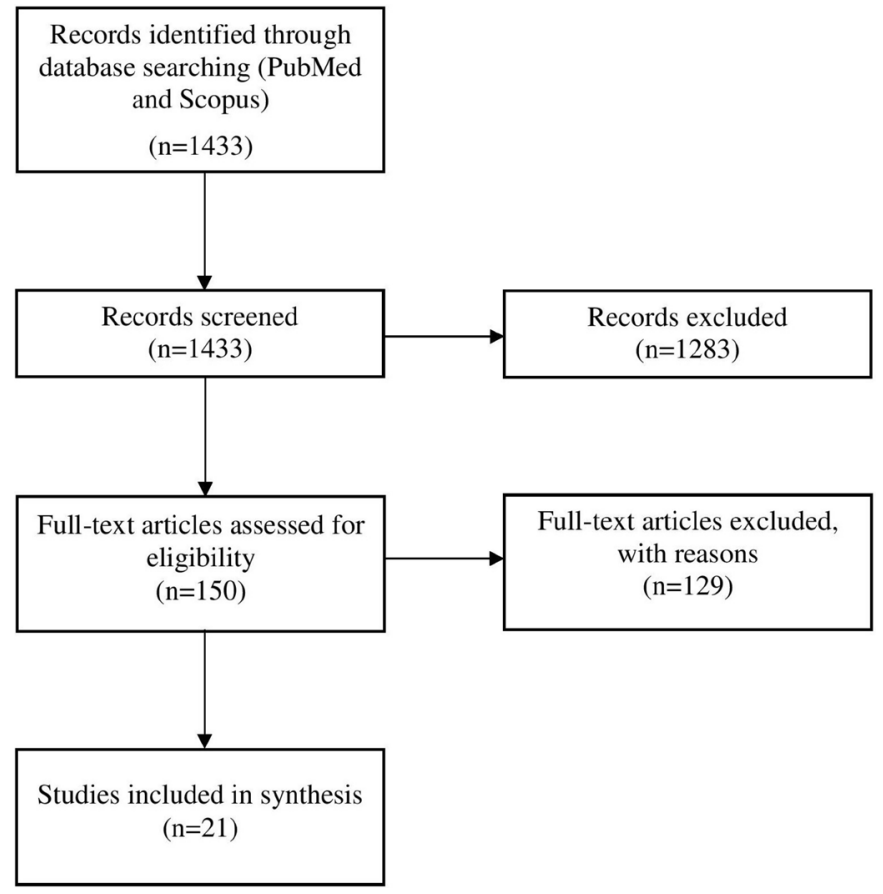

Figure 1 Preferred Reporting Items for Systematic Reviews and Meta-Analyses (PRISMA) flow chart of the literature review.

their experiences and insight on possible solutions (see online supplementary appendix 2 for interview questions). Interviewees were recruited using a convenience and snowball sampling. This means that social media, internal and external networks were used to directly recruit clinicians, especially those working in critical care, as alarms were most prevalent in critical care units. Additionally, interviewees were asked to suggest other potential interviewees who would be interested in participating in the study. To reduce sampling bias, interview results were to be presented to support, complement or confirm the results of the literature and grey literature reviews rather than as independent results. Each interview was started by informing interviewees with the background and aim of the study, why and how the interviewees were selected, and use of results and ethical considerations prior to starting the interview questions (online supplementary appendix 2). Participation was voluntary. Twenty-six clinicians from 10 countries were individually interviewed (online supplementary appendix 3). Verbal consent was given prior to the interviews by all interviewees. Each interview took approximately $30 \mathrm{~min}$. Six interviewees stated that they were involved in improvement work for alarm safety. Interview responses were noted down by the interviewers contemporaneously. The interviewers confirmed their notes to interviewees at the end of each interview to preserve and confirm interviewees' points of view. Interview responses were anonymised and treated as highly confidential.

Interview responses were collated and content analysis was conducted to extract significant words or phrases from the interview notes. ${ }^{37}$ The meaning of each significant word or phrase was defined and organised into themes using the reference themes of the improvement elements. A set of meetings were held among the authors to discuss extracted themes and their meanings. Any disagreements related to extracted themes and their meanings were solved by team consensus.

\section{The review of alarm-related standards}

Three alarm-related standards recognised by the FDA in the USA were included as the most commonly used standards for alarms of medical devices, namely IEC (International Electrotechnical Commission) 60601-1-8, ${ }^{1}$ IEC 62366-1:2015 ${ }^{38}$ and ANSI (American National Standards Institute)/AAMI HE 75:2009/®2013 ${ }^{39}$ (online supplementary appendix 4). Common themes of the improvement elements identified in the standards were clustered together according to the 10 reference themes of improvement elements. In addition, common themes were compared between the standards to identify gaps.

\section{RESULTS}

Results of the literature and grey literature reviews are presented first because the identified improvement elements are used as the reference themes for results of the interviews and the review of the alarm-related standards.

\section{The literature and grey literature reviews and interviews}

Twenty-one articles and seven publications on alarm quality improvement work were included in the literature and grey literature reviews (online supplementary appendix $1)$. The reviews identified 10 themes of improvement elements (table 1 and online supplementary appendix $5)$ to tackle alarm problems (see online supplementary appendix 6 for an overview of alarm problems by theme). Each improvement element was categorised as either a human, organisational or technical factor. Improvement elements that were predominantly related to improving staff interactions with alarms were categorised as human factors. Elements predominantly related to improving the processes of dealing with alarms in an environment or organisation were categorised as organisational factors. Elements predominantly related to improving alarms as equipment or a tool were categorised as technical factors. Importantly, nine reference themes of improvement elements were identified in the interview responses (table 1 and online supplementary appendix 7 ).

\section{Technical factors}

\section{Machine learning}

Machine learning was reported in the literature as improving alarm safety by, for example, developing smart alarms, ${ }^{16}{ }^{40}$ which were easy to integrate into existing systems ${ }^{41}$ to analyse multiple clinical data input of multiple patients, ${ }^{1742} 43$ and to decide whether staff actions or clinical interventions were needed for specific patients. ${ }^{43-45}$ If staff actions were needed, smart alarms would inform possible clinical risks of the condition and 


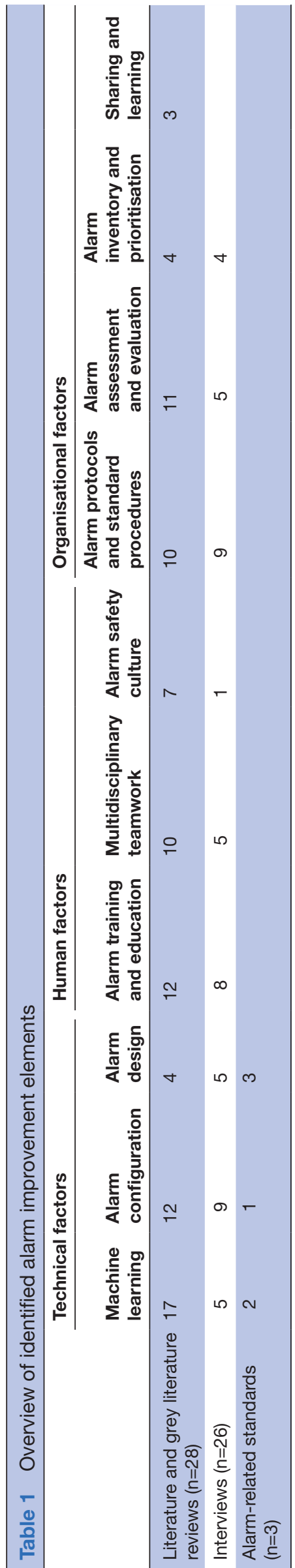

delivered this information to an appropriate, available staff member. ${ }^{17} 46-48$ This could be done by using multiple alarm signal notification technology when necessary ${ }^{49}$ or using appropriate technology such as pagers and cell phones. ${ }^{10} 4950$

Similarly, five interviewees suggested that hospitals should use more smart alarms to reduce false and non-actionable alarms and to direct staff attention to clinically critical alarms by, for example, using a centralised 'dashboard'. Interviewees also believed that smart alarms could connect multiple patient data or vital signs to predict and prevent critical situations by sending alarms to the appropriate staff for actions.

\section{Alarm configuration}

Literature suggested reconfiguring alarm settings to actionable levels as an effective solution to reducing false alarms. This could be done by (1) individualising alarm thresholds to patient-specific conditions or narrowing the thresholds, ${ }^{10} 46475152$ (2) setting alarms based on defined urgency from non-actionable to actionable alarms ${ }^{47} 49$ 52-54 and (3) eliminating non-actionable alarms or changing these alarms to vibration mode. ${ }^{49} 53 \quad 55 \quad 56$ Importantly, incorporating delays prior to firing alarms, ${ }^{17} 47$ suspending alarms temporarily prior to patient or staff manipulation, ${ }^{17}$ eliminating the self-resetting function in alarms ${ }^{53}$ or standardising alarm defaults across relevant units, departments or areas were suggested as an effective improvement effort. ${ }^{53}$

Interviewees perceived that the process of resetting or modifying alarm settings as too variable, non-standardised and cumbersome. This perception led to staff confusion and frustration. Interviewees suggested to involve front-line staff who dealt with alarms daily in redesigning alarm settings to be more user-friendly and fit-for-purpose.

\section{Alarm design}

The studies generally focused on improving alarm design for end users. For example, in a study by Seagull $e t a l^{34}$ additional informative patient vital signs were displayed on auditory displays to update staff with patient current states and to improve the informativeness of alarms. In addition, auditory signals were broadcasted only when there was an actionable change in patient status. Bennett et $a l^{33}$ focused on standardising alarm sounds based on perceptions of urgency. The study findings suggest that aperiodic or fluctuating composition of alarm tones or melodies were perceived as more urgent than other compositions.

Similarly, interviewees perceived that alarm melodies were not easy to discriminate and prioritise based on their urgency levels. Consequently, some alarms were being switched off to reduce alarm frequency but not being switched on again by mistake. Setting different levels of tones based on the urgency and severity of an alarm condition was suggested as a possible solution to indiscriminate alarm melodies. Interviewees also suggested 
that non-auditory alarms should be not be used independently but in addition to auditory alarms.

\section{Human factors}

\section{Alarm training and education}

Literature suggested that there was a lack of or limited training for alarm end users around alarm systems and alarm safety. ${ }^{10175357}$ The included studies focused on training and education on configuring alarms, for example, individualising and modifying alarm parameter defaults, ${ }^{1048} 52$ or eliminating false alarms and appropriately adjusting alarms in real time. ${ }^{53}$ Some studies integrated interactive educational activities as part of alarm intervention such as during daily stand-ups ${ }^{48}$ or clinical rounds. ${ }^{54}$ Several studies suggested the importance to conduct alarm training programmes prior to implementing new devices and continuously in addressing staff needs in dealing with alarming medical devices in the clinical environment where the devices were used. ${ }^{10} 1752$

Similarly, interviewees stated that healthcare staff did not necessarily have training in dealing with alarm problems and achieving alarm safety, which was perceived as crucial for patient safety. Even if there was training at all, it was usually not systematic or regular.

\section{Multidisciplinary teamwork}

The literature pinpointed that healthcare might be lacking understanding and conformity in tackling excessive alarm frequency in different clinical environments. ${ }^{57}$ Therefore, forming a multidisciplinary team to tackle alarm problems was named as being crucial for managing alarm systems. ${ }^{10} 17464849535456-58$ The studies suggested that the multidisciplinary team could consist of managers, front-line staff, clinical engineers, biomedical technicians, information technology and information system experts, patient safety experts, researchers, human factors engineers, administrative and facility staff, and even representatives from manufacturers of alarming medical devices. Representation from all environments where alarms were located was also crucial because different environments were likely to need different solutions.

Similarly, interviewees perceived that there was generally a lack of alarm accountability. Interviewees stated that it was crucial to involve staff from different areas encompassed in the patient journey to plan and solve alarm problems together.

\section{Alarm safety culture}

The literature suggested the importance of promoting alarm safety culture and involvement of senior manager for alarm safety. ${ }^{4856}$ For example, Ursprung et $a \check{\varphi}^{59}$ found that a blame-free culture facilitated acceptance by staff when implementing new interventions. In particular, engaging staff in identifying alarm problems and developing and implementing alarm solutions was suggested as a crucial element to promoting alarm safety culture. ${ }^{495657}$ There was one interviewee who mentioned the need to raise awareness of the importance of alarms if alarm safety was to be improved.

\section{Organisational factors \\ Alarm protocols and standard procedures}

Ten studies highlighted the fact that lack of alarm protocols, agreements and standard procedures on how to deal with alarms led to variable staff behaviours towards alarms and challenges in the workflow. ${ }^{1732}$ The literature suggested different focus areas including

- Establishing protocols to set patient-specific alarms. ${ }^{32}$

- Documenting alarm parameters in the medical records to improve alarm adjustment compliance. ${ }^{17}$

- Agreeing on procedures to pause or silence alarms to remove unnecessary alarms especially during patient or staff manipulation. ${ }^{32} 52$

- Standard procedures to ensure safe alarm management and response. ${ }^{46}$

- Adjusting staffing models that consider alarm response time as a primary task. ${ }^{17}$

- Reinforcing proper skin preparation for ECG leads and electrodes and daily replacement electrode to ensure proper signal acquisition and reduce artefacts. 1748495253

- Ensuring accountability of all alarms in an environment. $^{1052}$

Establishing an evaluation protocol was seen as necessary to measure the effect of the established alarm protocols or standard procedures. ${ }^{52}$

Similarly, interviewees felt that it was crucial to establish alarm protocols and standard procedures to give sufficient guidance to staff in tackling alarm problems. Staff involvement in developing and establishing alarm protocols and standard procedures was said as key to compliance.

\section{Alarm assessment and evaluation}

Alarm assessment and evaluation were suggested to be conducted prior to and after adopting an alarming medical device. ${ }^{559}$ The assessment and evaluation were suggested to be conducted in a clinical environment and to check alarm types, audibility, validity and relevance. ${ }^{1017}$ Factors related to the environment and organisations where the alarms were located such as bed to alarm ratio, purpose and length of alarm conditions, staffing levels, high-risk patient ratio required intense monitoring, unit layout and background noise were also considered important. ${ }^{49557}$ Measurement of staff response time and how staff responded to alarm signals was suggested to help to understand inconsistent staff behaviour in responding to alarm signals and use measurement results for data analysis. ${ }^{5758}$ Results of the assessment and evaluation could generate alarm data for improvement. ${ }^{48} 49$

Interviewees emphasised the importance of an evaluation to measure effects after implementation of alarm intervention. This was because some intervention or strategies might be ineffective or could even cause new problems. 


\section{Alarm inventory and prioritisation}

Literature pinpointed that sources of alarms were not always successfully identified by staff, increasing the risk of ignorance of critically clinical alarms. ${ }^{32}$ In addition, many alarm conditions were duplicated, increasing alarm frequency unnecessarily. ${ }^{49}$ Accordingly, prioritising actionable alarm signals was suggested as key to reduce excessive alarm signals ${ }^{32}$ by conducting an alarm inventory to gather data such as the number of alarms per bed, their types and duration, and documenting baseline alarm conditions and signals. ${ }^{49}$ Prioritisation could also mean subordinating or, if necessary, eliminating lower-priority warning or advisory alarm conditions to, for example, visual signals or vibration.

Interviewees, similarly, perceived that it was important to be familiar with all alarms and their purpose in an environment so that alarms could be prioritised based on urgency levels.

\section{Sharing and learning}

Vockley et $a \tilde{l}^{\tilde{7}}$ suggested that an organisation might have limited knowledge around alarm systems, technologies, policies, protocols and manufacturers. Alarm intervention thus should include organisational activities to seek out, visit or consult other healthcare systems or organisations to gather knowledge in managing alarm systems and achieving alarm safety. Encouraging organisational learning was also key for alarm safety. This could be conducted by looking for commonalities of alarm problems and solutions across units and departments.

\section{The review of alarm-related standards}

The three standards were intended to be used by manufacturers of alarming medical devices and thus posed a different focus compared with the improvement elements found in the reviews and interviews. Consequently, the findings were reported in this independent section instead of combining them with the findings of the literature and grey literature reviews and the interviews. The findings show that the three standards discussed alarm design, machine learning and alarm configuration (table 2 and online supplementary appendix 8). In subthemes, there were only a few improvement elements covered in more than one standard, suggesting the three standards were complementary to each other. See online supplementary appendix 8 for example content of standards.

\section{DISCUSSION}

This study identifies 10 themes of improvement elements for alarm safety (table 1). The improvement elements found are comparable to the recommendations to improve alarm safety suggested by ECRI, ${ }^{60} \mathrm{AAMI}^{2}$ and the Joint Commission. ${ }^{61}$ These recommendations also emphasise the importance of multidisciplinary teamwork and leadership as well as staff engagement. Moreover, alarm data and assessment, alarm inventory, strategies to address alarm issues, alarm configuration and settings, alarm training and sharing of learning are highlighted in the recommendations. The findings also show that several improvement elements were explored and investigated more than others such as machine learning in the literature, whereas interviewees mentioned alarm configuration most often to improve alarm safety. Nevertheless, the most discussed improvement elements may not necessarily represent the most important ones for improving alarm safety. This is because improving alarm safety requires implementation of improvement efforts that address human, organisational and technical issues as an integrated approach. ${ }^{2122347}$ For example, machine learning is a technical solution to decentralised alarms that makes sure alarms notify mobile staff. However, implementing machine learning alone cannot necessarily address issues such as inconsistent staff responses to alarms, a human factor. Similarly, to implement alarm configuration as a technical solution, an organisation also needs to educate and train their staff to set appropriate alarm thresholds, an organisational factor. Nevertheless, the order in which improvement elements can be implemented may not be arbitrary.

We propose a step-by-step guide to optimise implementation of the improvement elements specific for the hospital setting or a clinical environment whose staff deal with alarms daily (figure 2). The formation of a multidisciplinary team is crucial and should ideally be in place prior to implementation of any other improvement elements (step 1), as also suggested by $\mathrm{AAMI}^{2}$ and ECRI. ${ }^{60}$ The four improvement elements in step 2 should be seen as equivalent in implementation order, such that they can be implemented either simultaneously or after one another depending on the need in a clinical environment. For example, one department could promote alarm safety culture by involving staff in establishing alarm protocols and standard procedures, whereas another department that has regular audits may want to initially integrate alarm assessment and evaluation into the audits to identify gaps prior to promoting alarm safety culture and establishing alarm protocols and standard procedures. The two improvement elements in step 3 can be implemented after the four improvement elements on step two are implemented. After concluding steps 1-3, step 4 can be used as a checkpoint to assess whether the implemented improvement elements have positively influenced alarm safety. Any learning should be shared and used to improve the intervention (step 5), emphasising continuous quality improvement. ${ }^{24} 6061$

The improvement elements of alarm design and machine learning were not included in the guide because improving alarm design and, to a certain extent, use of machine learning are likely to require more active participation from manufacturers and other alarm stakeholders such as regulators. Similarly, the two improvement elements are not emphasised in the top key suggestions to improve alarm safety by ECRI, ${ }^{60} \mathrm{AAMI}^{2}$ and the Joint Commission. ${ }^{61}$ Nevertheless, we encourage alarm end users and manufacturers to work closely to improve alarm design for specific alarming devices by, 
Table 2 Improvement elements covered in the alarm-related standards

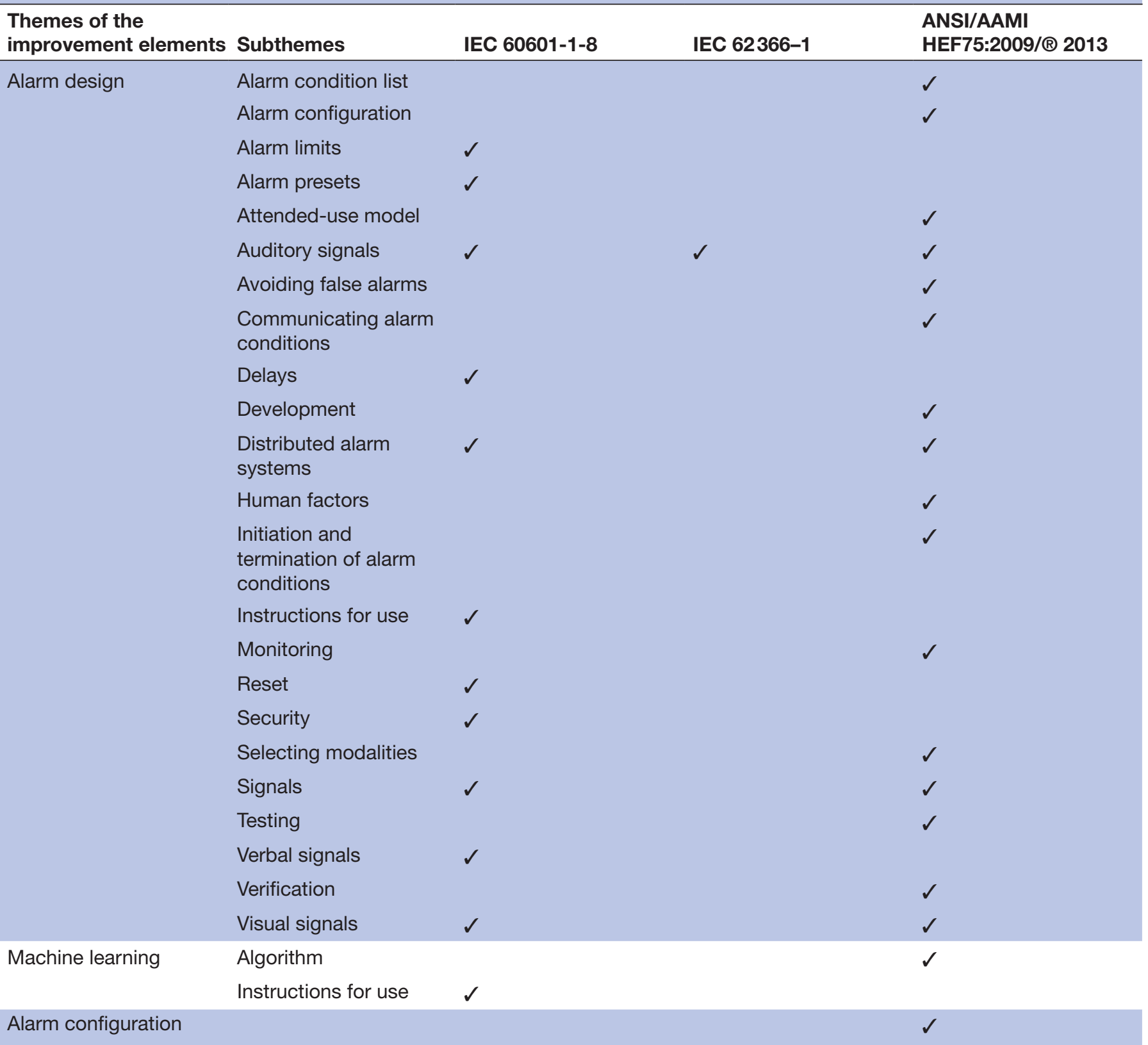

for example, including additional patient vital signs to make alarms more informative, ${ }^{34}$ or standardising alarm sounds of different devices based on end users' perceived urgency to improve alarm discrimination. ${ }^{33}$ Similarly, the use of machine learning is also encouraged to improve alarm safety by developing real-time smart alarms capable of analysing multiple clinical data input for multiple patients. The alarms should be relatively easy to integrate into existing systems and capable of deciding whether actions are needed for specific patients, and if so, sending the alarms to appropriate, available staff.

Although the main focus of all three alarm-related standards was alarm design (table 2), findings of the reviews and interviews showed that when different medical devices were placed in a clinical environment, staff struggled to integrate different alarm systems due to devices' differences in the functions, characteristics and alarm settings modification. ${ }^{48}$ This shows a gap and opportunities for future alarm design, with a clear need for manufacturers and regulators to work together with end users to create more standardised, effective alarm systems across different medical devices.

Our study is not without limitations. First, the literature reviews did not review the impact of improvement elements. This was because the impact measures differed greatly between studies and between improvement elements, if at all reported. If we had excluded studies that omitted impact measures, the study would have been too limited to achieve its objective of providing an overview of documented, demonstrated alarm improvement elements. Second, interviewees were recruited through convenience and snowball sampling that might cause 


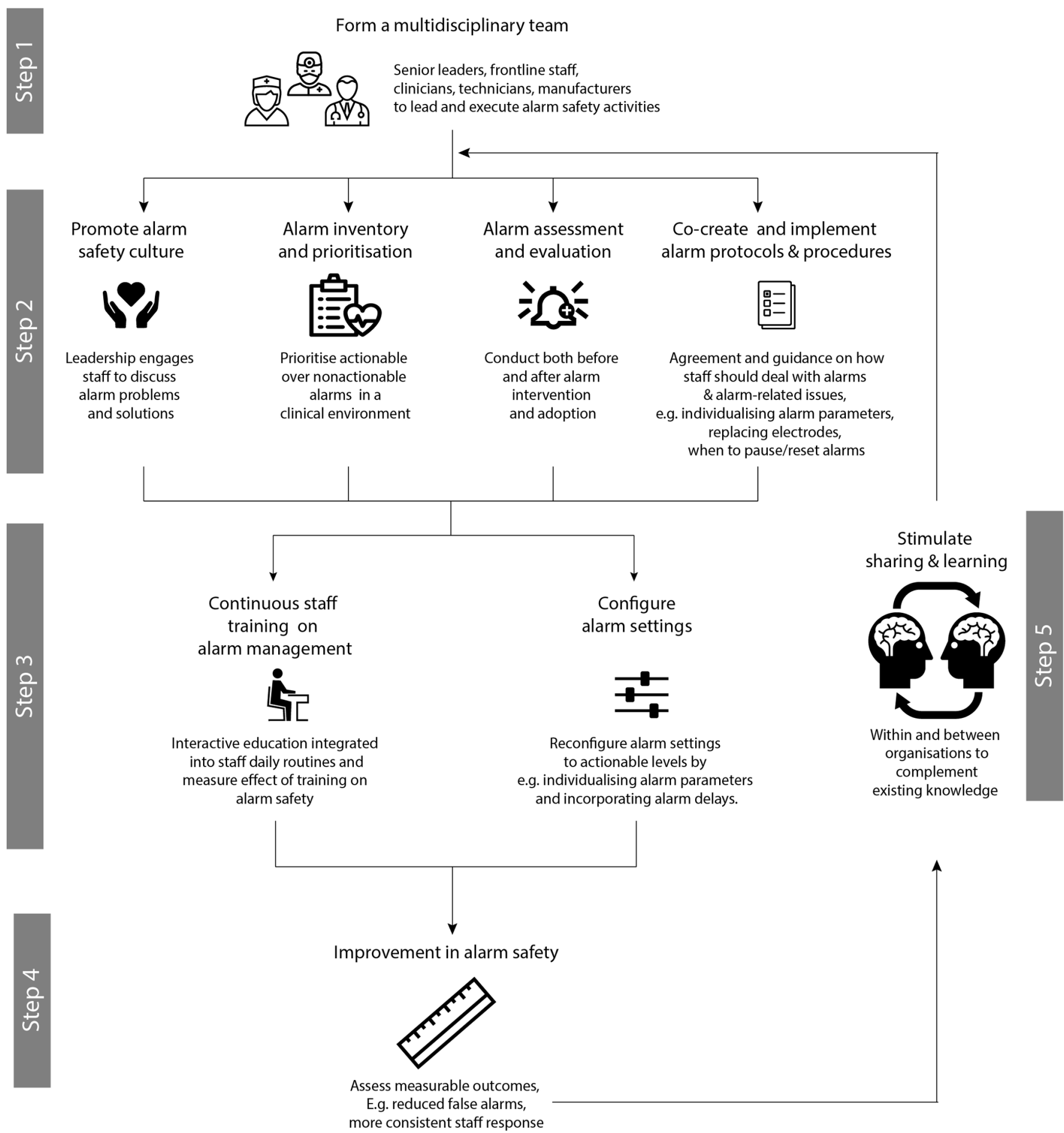

Figure 2 Proposed step-by-step guide to optimise implementation of the alarm improvement elements in healthcare.

sampling bias. Consequently, the interview findings were used to support, complement or confirm the findings of the literature reviews rather than as independent findings. The representation of 10 countries in the interview sample may limit the study results for generalisations to other locations. Finally, although the three alarm-related standards are the most common ones used by alarm manufacturers, the study did not include other standards, which may limit the findings.

\section{CONCLUSION}

Improving alarm safety can be achieved by addressing and incorporating not only the technical factor of alarms but also human and organisational factors in an integrated approach. There is still a gap between alarm-related standards and how the standards are translated into practice, especially in a clinical environment that uses multiple alarming medical devices from different manufacturers. Standardisation across devices and manufacturers and 
the use of machine learning in improving alarm safety should be discussed in future collaboration between alarm manufacturers, end users and regulators.

Acknowledgements The authors thank Stephen Leyshon for his initial input to the project planning and for comments on an earlier draft of this manuscript. They also thank Dr Sharmini Alagaratnam for critically reviewing the manuscript.

Contributors TAB initiated, planned and managed the study. All authors conducted the literature and the grey literature reviews, interviews, the review of the alarmrelated standards and performed the analyses. All authors wrote and reviewed the manuscript.

Competing interests None declared.

Provenance and peer review Not commissioned; externally peer reviewed.

Data sharing statement № additional data are available.

Open access This is an open access article distributed in accordance with the Creative Commons Attribution Non Commercial (CC BY-NC 4.0) license, which permits others to distribute, remix, adapt, build upon this work non-commercially, and license their derivative works on different terms, provided the original work is properly cited, appropriate credit is given, any changes made indicated, and the use is non-commercial. See: http://creativecommons.org/licenses/by-nc/4.0/.

(C) Published by the BMJ Publishing Group Limited. For permission to use (where not already granted under a licence) please go to http://www.bmj.com/company/ products-services/rights-and-licensing/

\section{REFERENCES}

1. International Electrotechnical Commission. IEC 60601-1-8 Medical electrical equipment - Part 1-8: General requirements for basic safety and essential performance. Collateral Standard: General requirements, tests and guidance for alarm systems in medical electrical equipment and medical electrical systems. Geneva, Switzerland: International Electrotechnical Commission, 2012.

2. AAMI Foundation. Clinical alarm: 2011 Summit convened by AAMI, FDA, TJA, ACCE and ECRI Institute. 2011 http://s3.amazonaws.com/ rdcms-aami/files/production/public/FileDownloads/Summits/2011 Alarms_Summit_publication.pdf2016.

3. ECRI Institute. ECRI Institute Announces Top 10 Health Technology Hazards for 2015. https://www.ecri.org/press/Pages/ECRI-InstituteAnnounces-Top-10-Health-Technology-Hazards-for-2015.aspx2016.

4. Sendelbach S, Funk M. Alarm fatigue: a patient safety concern AACN Adv Crit Care 2013;24:378-86.

5. Kowalczyk L. Suit over cardiac monitor settled - MGH patient died despite alarms. Boston Globe 2011

6. Kowalczyk L. 'Alarm fatigue' a factor in $2 d$ death - UMass hospital cited for violations. Boston Globe 2011.

7. Drew BJ, Harris P, Zègre-Hemsey JK, et al. Insights into the problem of alarm fatigue with physiologic monitor devices: a comprehensive observational study of consecutive intensive care unit patients. PLoS One 2014;9:e110274.

8. FDA Patient Safety News: Show \#106, 2011. http://www.fda.gov/ downloads/Safety/FDAPatientSafetyNews/UCM417915.pdf2016.

9. Love LC, Millin CJ, Kerns CD. Take precautions with audible alarms on ventilators. Nursing2011;41:65.

10. Graham KC, Cvach M. Monitor alarm fatigue: standardizing use of physiological monitors and decreasing nuisance alarms. Am J Crit Care 2010;19:28-34

11. Bridi AC, da Silva RC, de Farias CC, et al. [Reaction time of a health care team to monitoring alarms in the intensive care unit: implications for the safety of seriously ill patients]. Rev Bras Ter Intensiva 2014;26:28-35

12. Kowalczyk L. State reports detail 11 patient deaths linked to alarm fatigue in Massachusetts. Boston Globe 2011.

13. Lawless ST. Crying wolf: false alarms in a pediatric intensive care unit. Crit Care Med 1994;22:981-5.

14. Chambrin MC, Ravaux P, Calvelo-Aros D, et al. Multicentric study of monitoring alarms in the adult intensive care unit (ICU): a descriptive analysis. Intensive Care Med 1999;25:1360-6.

15. Tsien CL, Fackler JC. Poor prognosis for existing monitors in the intensive care unit. Crit Care Med 1997;25:614-9.

16. Siebig $S$, Kuhls $S$, Imhoff $M$, et al. Intensive care unit alarms--how many do we need? Crit Care Med 2010;38:451-6.

17. Cvach M. Monitor alarm fatigue: an integrative review. Biomed Instrum Technol 2012;46:268-77.
18. Bonafide CP, Lin R, Zander M, et al. Association between exposure to nonactionable physiologic monitor alarms and response time in a children's hospital. J Hosp Med 2015;10:345-51.

19. AAMI Foundation. Human factors engineering-design of medical devices (ANSI/AAMI HE75) 2009/(R)2013. Arlington, VA: AAMI, 2013.

20. Edworthy J. Alarms are still a problem!. Anaesthesia 2013;68:791-4.

21. Bliss JP, Gilson RD, Deaton JE. Human probability matching behaviour in response to alarms of varying reliability. Ergonomics 1995;38:2300-12.

22. Edworthy J, Hellier E. Alarms and human behaviour: implications for medical alarms. Br J Anaesth 2006;97:12-17.

23. Xie H, Kang J, Mills GH. Clinical review: The impact of noise on patients' sleep and the effectiveness of noise reduction strategies in intensive care units. Crit Care 2009;13:13(2):1

24. AAMI Foundation. Clinical alarm management compendium. 2016;2015 http://s3.amazonaws.com/rdcms-aami/files/production/ public/FileDownloads/Foundation/Reports/Alarm_Compendium_ 2015.pdf2016.

25. ECRI Institute. Top 10 Health Technology Hazards for 2015: ECRI Institute, 2014.

26. ECRI Institute. Top 10 Health Technology Hazards for 2014: ECR Institute, 2013. Available from. https://www.ecri.org/Resources/ Whitepapers_and_reports/2014_Top_10_Hazards_Executive_Brief. pdf2016.

27. ECRI Institute. Top 10 Health Technology Hazards for 2013: ECR Institute, 2012. Available from. http://www.samtit.nu/filarkiv/nya filarkivet/sakerhet/health_devices_top_10_hazards_2013.pdf2016.

28. ECRI Institute. Top 10 Health Technology Hazards for 2012: ECRI Institute, 2011. Available from. http://www.samtit.nu/filarkiv/nya filarkivet/sakerhet/health_devices_top_10_hazards_2012.pdf2016.

29. The Joint Commission. The Joint Commission announces 2014 national patient safety goal, 2013. Available from. http://www. jointcommission.org/assets/1/18/jcp0713_announce_new_nspg. pdf2016.

30. Healthcare Technology Foundation. 2011 National Clinical Alarms Survey: Perceptions, Issues, Improvements, and Priorities of Healthcare Professionals: Healthcare Technology Foundation. 2011 http://www.thehtf.org/documents/2011_HTFAlarmsSurveyOverallRe sults.pdf2016.

31. Sowan AK, Gomez TM, Tarriela AF, et al. Changes in Default Alarm Settings and Standard In-Service are Insufficient to Improve Alarm Fatigue in an Intensive Care Unit: A Pilot Project. JMIR Hum Factors 2016;3:e1.

32. Applying an Evidence-based Approach to Managing Alarm Safety: A University Health Network Case Study.. World Congress on Medical Physics and Biomedical Engineering. Toronto, Canada: Springer, 2015:7-12.

33. Urgency Analysis of Audible Alarms in The Operating Room: ISMIR, 2011

34. Auditory alarms: from alerting to informing. Proceedings of the Human Factors and Ergonomics Society Annual Meeting: SAGE Publications, 2000

35. Siebig S, Sieben W, Kollmann F, et al. Users' opinions on intensive care unit alarms-a survey of German intensive care units. Anaesth Intensive Care 2009;37:112.

36. Regional Committees for Medical and Health Research Ethics. Examples of activities that require approval from REC 2012.

37. Yin RK. Case study research: Design and methods: sage, 2009.

38. International Electrotechnical Commission. IEC 62366-1:2015. Medical devices - Part 1: Application of usability engineering to medical devices, 2015

39. AAMI Foundation. ANSI/AAMI HE75:2009/(R)2013. Human factors engineering - Design of medical devices, 2013.

40. Incremental real time support vector machines for health monitoring system. Complex Systems (WCCS), 2014 Second World Conference on: IEEE, 2014

41. Bennett CL, McNeer RR. PT-SAFE: a software tool for development and annunciation of medical audible alarms. Anesth Analg 2012;114:576-83.

42. Medical signal processing in the ICU. 2007 IEEE 33rd Annual Northeast Bioengineering Conference: IEEE., 2007.

43. Real-time development of patient-specific alarm algorithms for critical care. 2007 29th Annual International Conference of the IEEE Engineering in Medicine and Biology Society: IEEE, 2007.

44. Real-time evaluation of patient monitoring algorithms for critical care at the bedside. 2007 29th Annual International Conference of the IEEE Engineering in Medicine and Biology Society: IEEE, 2007.

45. Reducing ECG alarm fatigue based on SQI analysis. Computing in Cardiology: IEEE, 2014. 
46. Ketko AK, Martin CM, Nemshak MA, et al. Balancing the Tension Between Hyperoxia Prevention and Alarm Fatigue in the NICU. Pediatrics 2015;136:e496-e504.

47. Paine CW, Goel VV, Ely E, et al. Systematic Review of Physiologic Monitor Alarm Characteristics and Pragmatic Interventions to Reduce Alarm Frequency. J Hosp Med 2016;11:136-44.

48. Epstein ML, Smith K, Snavely V. Safety Innovations - Fighting Alarm Fatigue with Data-Driven Interventions - The NCH Healthcare Device Eco-System Experience The AAMI Foundation. 2016 http://s3. amazonaws.com/rdcms-aami/files/production/public/FileDownloads/ Foundation/Safetylnnovation/2016_SI_NCH_Fighting_Alarm_Fatigue. pdf2016.

49. AAMI Foundation. Safety Innovation - Using Data to Drive Alarm System Improvement Efforts - The Johns Hopkins Hospital Experience: The AAMI Foundation and the Healthcare Technology Safety Institute. 2012 https://s3.amazonaws.com/rdcms-aami/files/ production/public/FileDownloads/HTSI/Johns_Hopkins_White_Paper. pdf2016.

50. Cvach MM, Frank RJ, Doyle P, et al. Use of pagers with an alarm escalation system to reduce cardiac monitor alarm signals. J Nurs Care Qual 2014;29:9-18.

51. Solsona JF, Altaba C, Maúll E, et al. Are auditory warnings in the intensive care unit properly adjusted? J Adv Nurs 2001;35:402-6.

52. Allen JS, Hileman K, Ward A. Safety Innovations - Simple Solutions for Improving Patient Safety: In. Monitoring C, Cardiac Monitoring - Eight Critical Elements to Monitor Alarm Competency - University of Pittsburgh Medical Center (UPMC): Presbyterian Hospital: the AAMI Foundation and the Healthcare Technology Safety Institute, 2012. http://s3.amazonaws.com/rdcms-aami/files/production/public/ FileDownloads/HTSI/Alarm_Competency\%20_White_Paper.pdf2016.

53. Whalen DA, Covelle PM, Piepenbrink JC, et al. Novel approach to cardiac alarm management on telemetry units. $J$ Cardiovasc Nurs 2014;29:E13-E22.

54. AAMI Foundation. Safety Innovations - Safeguarding Patients With Surveillance Monitoring - The Dartmouth-Hitchcock Medical Center
Experience the AAMI Foundation and the Healthcare Technology

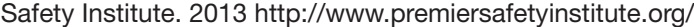
wp-content/uploads/Safeguarding-patients-with-surveillancemonitoring-Dartmouth-Hitchcock.pdf2016.

55. Khuntia J, Tanniru M, Weiner J. Juggling digitization and technostress: The case of alert fatigues in the patient care system implementation. Health Policy and Technology 2015;4:364-77.

56. Lipschultz A. Safety Innovations - Clinical Practice Changes Associated with Alarm Standardization - The Boston Medical Center Experience the AAMI Foundation and the Healthcare Technology Safety Institute;. 2014 http://s3.amazonaws.com/rdcms-aami/files/ production/public/FileDownloads/HTSI/Alarm_Standardization_ White_Paper_2.pdf2016.

57. Vockley M. Safety Innovations - Recommendations for alarm signal standardization and more innovation - The Christiania Care Health System Experience: the AAMI Foundation and the Healthcare Technology Safety Institute;. 2012 http://www.premiersafetyins titute.org/wp-content/uploads/Christiana-Care-Alarm-Signal. pdf2016.

58. Talley LB, Hopper J, Jacobs B, et al. Safety Innovations Cardiopulmonary Monitors And Clinically Significant Events in Critically III Children - Children's National Medical Center: the AAMI Foundation and the Healthcare Technology Safety Institute. $2013 \mathrm{http}: / / \mathrm{s} 3$.amazonaws.com/rdcms-aami/files/production/ public/FileDownloads/Foundation/Safetylnnovation/2013_SI_ Cardiopulmonary Monitors Childrens.pdf2016.

59. Ursprung R, Gray JE, Edwards WH, et al. Real time patient safety audits: improving safety every day. Qual Saf Health Care 2005;14:284-9.

60. ECRI Institute. Strategies to improve monitor alarm safety: ECRI Institute, 2011. https://www.ecri.org/Resources/Whitepapers_and reports/Monitor_Alarm_Safety_Poster_Presentation.pdf.

61. The Joint Commission. Medical device alarm safety in hospitals: Sentinel event alert/Joint Commission on Accreditation of Healthcare Organizations, 2013. 